Aus der Hamburgischen Staatsirrenanstalt Langenhorn (Dir. Prof. Dr. Neuberger).

\title{
Über eine Methode der objektiven Darstellung der Loka- lisation von multiplen Herden im Gehirn. \\ Von
}

Dr. med. et phil. Ernst Bischoff.

(Mit 2 Abbildungen.)

Anlass zur Anwendung und Ausarbeitung der Methode gab ein Fall von Gehirncarcinom mit zahlreichen Metastasen. Der Fall ist ausführlicher beschrieben in der Deutschen Zeitschrift für Nervenheilkunde 54. Band, 6. Heft, Seite 415. Die gewöhnliche Art der Darstellung der Lokalisation der Hirngeschwülste ist diejenige der wörtlichen Beschreibung der Verhältnisse der Umgebung des Herdes. Dabei wird diese Umgebung bezeichnet mit den entsprechenden anatomischen Namen, z. B. Tumor im mittleren Drittel der rechten Zentralwindung, oder mehr mit Bezugnahme auf physiologische Verhältnisse Tumor im Mark der Seestrahlung usw. Diese Bezeichnung gibt natiurlich die Lage meistens in befriedigender Weise wieder, aber das doch nicht immer, da die Nomenklatur der einzelnen Teile des Gehirns nicht immer genügend weitgehend durchgearbeitet ist, so dass die einzelnen Teile so differenziert sind, dass sie obne weiteres mit makroskopischer oder auch mikroskopischer Methode identifiziert werden können. Andererseits lässt diese Methode vieles zu wünschen übrig bezüglich ihrer Anschaulichkeit, und kann es schon nicht ganz leicht sein, sich ein genaues Bild über die Lage eines einzelnen Tumors zu machen, so ist es sehr schwer, zahlreiche grössere oder kleinere Herde so iibersichtlich in ihrer Lage zu beschreiben, dass man sie mit genügender Klarheit und Deutlichkeit vor Augen hat.

Dazu kommt der Spielraum, den man dem subjektiven Faktor z. B. bei der Auswahl derjenigen Teile lassen muss, der die Umgebung charakterisieren soll.

An Stelle dieser logisch sprachlichen Methode wählt die hier geschilderte Methode die optische Darstellung im Bilde, die natürlich auch schon früher die alte erwähnte Methode ergänzte. Das Neue 
liegt hier vielmehr in der systematischen Anordnung der Bilder. Dabei wird zur Zeichnung die objektiv arbeitende Photographie gewählt und man bekommt so die Möglichkeit einer objektiven Darstellung der Lage des oder der Herde.

Das Gehirn wird im ganzen herausgenommen. Beabsichtigt man Untersuchungen mit Methoden zu machen, z. B. nach Nissl, die eine möglichst schnelle Fixierung kleiner Teile erfordert; so würde man das Gehirn sofort photographieren: von oben und von der Seite, in bestimmten Fällen auch in entsprechend anderer Ansicht. Dann schneidet man die betreffenden Teilchen heraus und behandelt lege artis. Nun wird das ganze Gehirn für einige Tage in starkem Formol gehärtet und kommt dann in Alkohol. Nach etwa 6-8 Wochen kann man an den wünschenswerten Stellen Frontalschnitte machen. Diese Frontalschnitte ( $1-f 7$ ) werden einzeln photographiert und untereinander im Bilde angeordnet. In der Photographie kann man gegebenenfalls die Tumorstellen durch Schraffierung und Ähnliches deutlich heryorbeben, wenn nötig mit Zahlen versehen (1-9).

Die Frontalschnitte werden dann in ihrer früheren Anordnung aneinandergelegt und das Gehirn wird jetzt in der Ansicht von oben photographiert. $\left.{ }^{1}\right)$ In dieser Photographie werden die Einzelschnitte auch z. B. mit $f$ (frontal) und fortlaufenden Zahlen versehen, wie die photographischen Bilder der Flächen die entsprechende Bezeichnung erhalten haben.

Nun werden die Frontalschnitte mit einer starken konzentrierten, nach Möglichkeit sehr dickflüssigen Celloidinlösung für die Einbettung mikroskopischer Präparate übergossen und aufeinander gelegt. Sie kleben jetzt aber wenig intensiv zusammen. Um dem Ganzen die genügende Festigkeit zu geben, begiesst man das ganze Gehirn nach einigen Minuten mit einer dünneren Celloidinlösung, wie sie für das Einbetten der mikroskopischen Präparate verwandt wird. Sehr bald ist von dieser Lösung so viel verdunstet, dass sie eine gut zusammenhängende Schicht über dem ganzen Gehirn bildet und der Masse einen Zusammenhang gibt, der sie für die weitere Behandlung geeignet macht.

Es werden jetzt an diesem wieder völlig zusammenhängend erscheinenden Gehirn Sagittalschnitte gemacht. Auch diese wieder werden photographiert und etwa mit s (sagittal) und fortlaufenden Ziffern versehen. Die Herdstellen der Frontalschnitte werden jetzt an den Sagittalschnitten an entsprechenden Stellen sichtbar werden. Man

1) event. überflüssig, deshalb hier in der Tafel nicht besonders angegeben, vgl. Fig. 1.

Deutsche Zeitschrift f. Nervenheilkunde. Bd. 55 . 

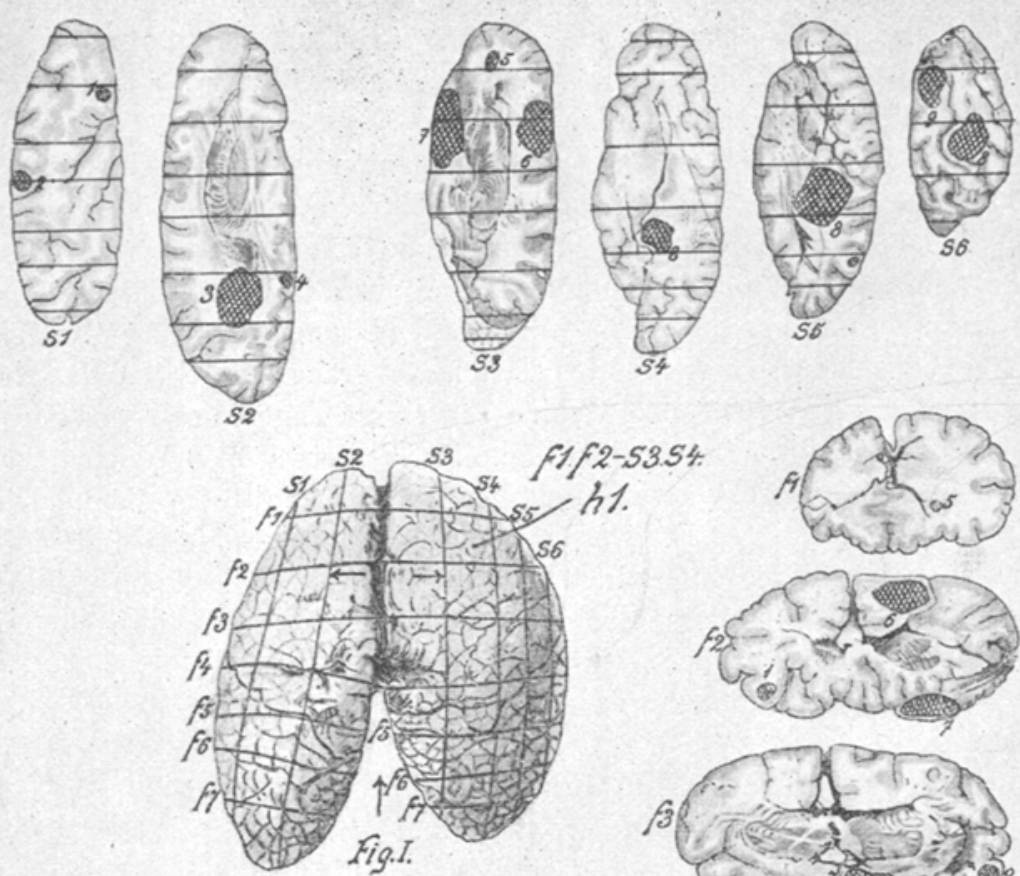

की की के

c1. $2-53.54$
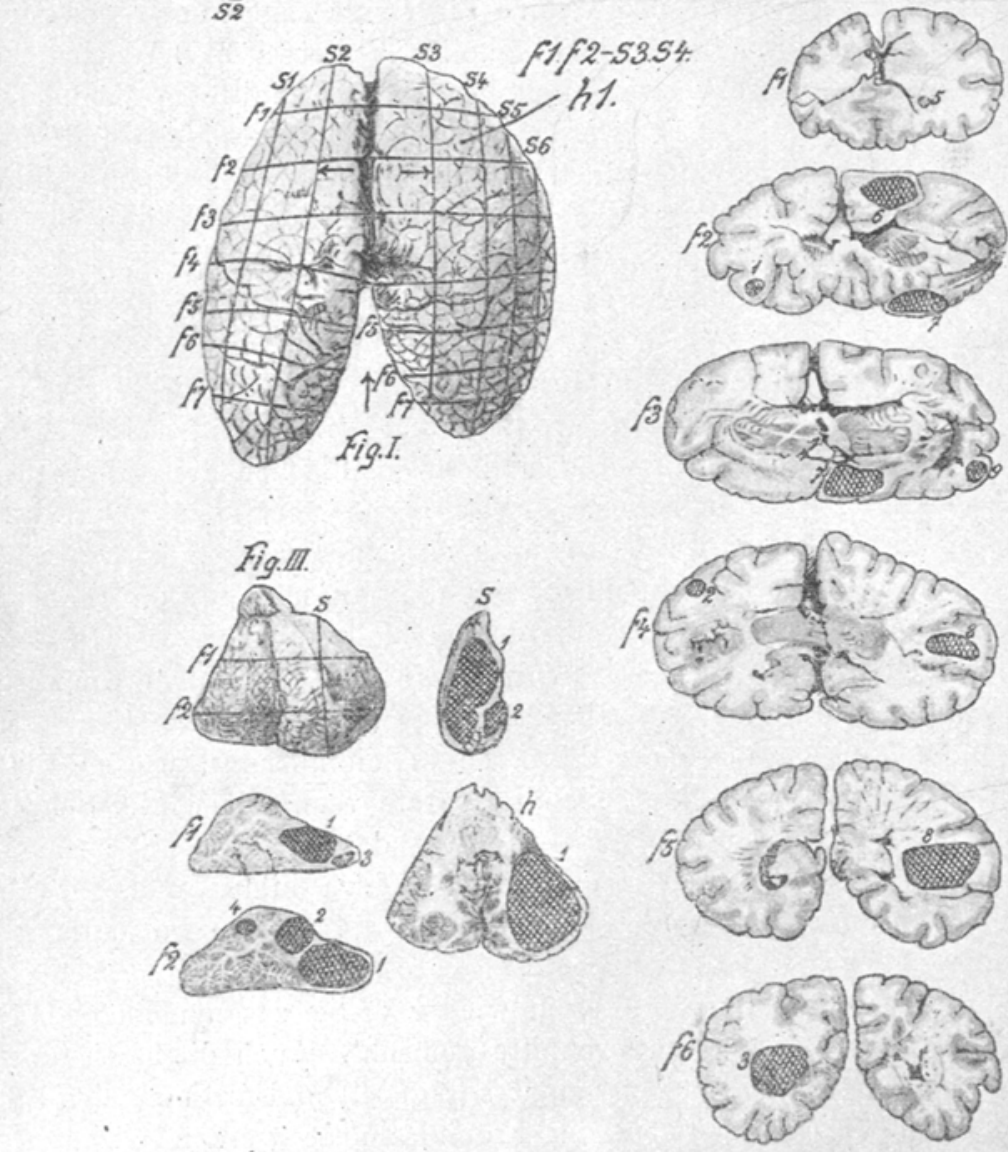

- Tumor: 
Über eine Methode der objektiven Darstellung der Lokalisation usw.

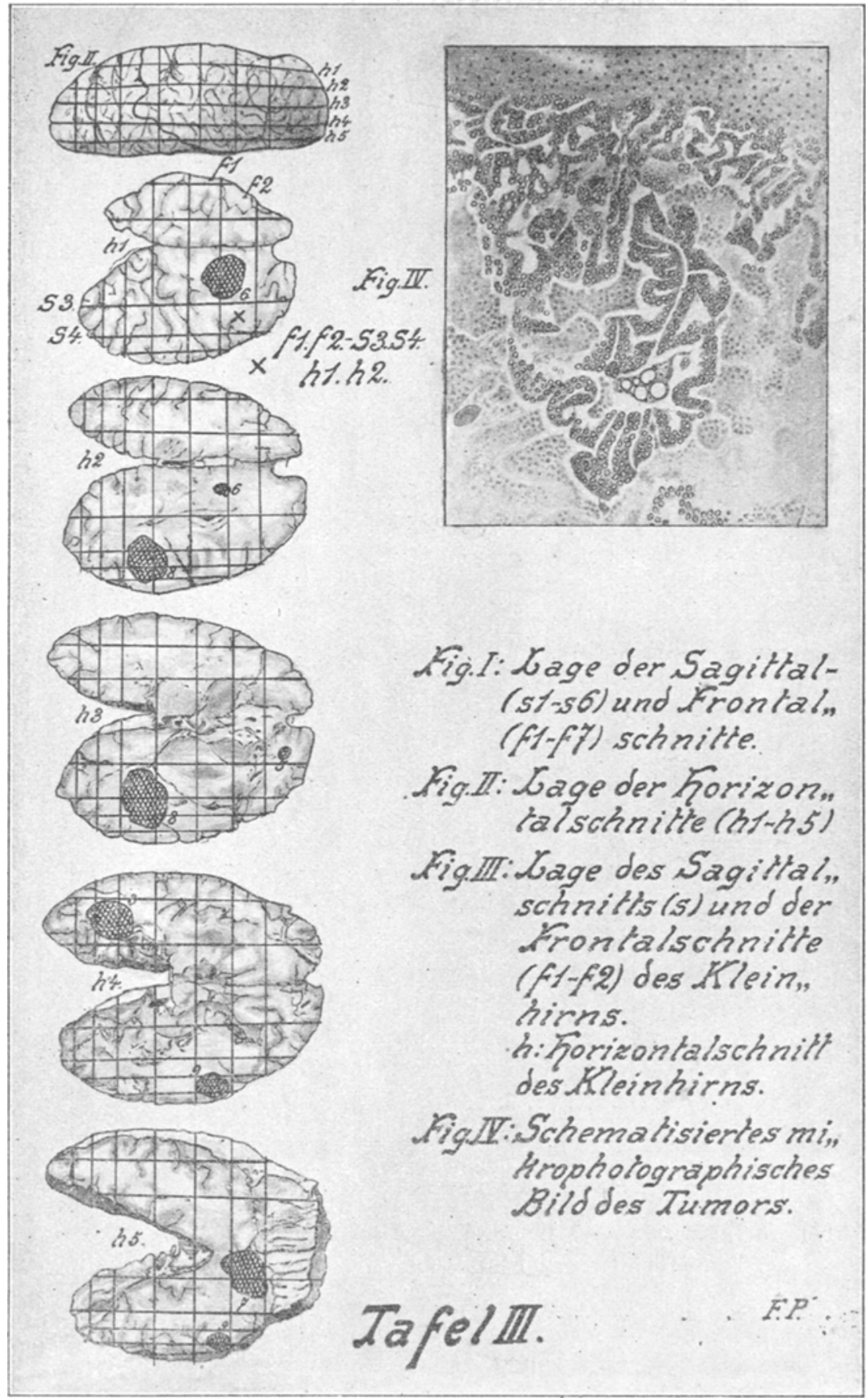


kann sie so auf der Photographie mit den entsprechenden Ziffern der Tumorstellen der Frontalschnitte bezeichnen.

Nachdem die Sagittalschnitte wieder mit der stark konzentrierten Celloidinlösung zusammengeklebt sind und das Gehirn wieder mit der dïnneren Celloidinlösung zu einer zusammenhängenden Masse gemacht worden ist, und in der Ansicht von oben (Fig. 1) photographiert worden, legt man die gew ünschte Zahl von Horizontalschnitten an. Diese Horizontalschnitte werden ebenso behandelt wie die Frontalschnitte und Sagittalschnitte und die Herdbilder in entsprechender Weise nummeriert.

Dann werden auch die Horizontalschnitte aufeinander geklebt und das Gehirn wieder mit der dünnen Celloidinlösung übergossen. Es zeigt auch jetzt in der Oberfläche kaum irgendwelche Änderungen als die erwähnten Schnittlinien.

Jetzt wird das Gehirn von der Seite photographiert.

Auf diesen Photographien sieht man dentlich den Verlauf der einzelnen Schnittlinien, die man an diesem Übersichtsbilde entsprechend bezeichnet (Fig. 2).

Um ein Beispiel als Darstellung der Methode zu geben, sei das Bild des erwähnten Falles wiedergegeben.

Abbildung: Seite 50 und 51.

Auf der Tafel ist auch das Kleinhirn in entsprechender Weise behandelt dargestellt.

Die so behandelten Stücke sind durchaus noch geeignet für eine weitere histologische Untersuchung, soweit ein in toto gehärtetes Gehirn für eine solche überhaupt noch in Frage kommt:

Für diese Untersuchung aber eröffnet sich dann noch die Möglichkeit einer bequemen und deutlichen Bezeichnung der betreffenden Stücke, von denen die Schnitte stammen, indem einfach auf dem einzelnen Schnittbilde die betreffende Stelle bezeichnet wird.(x) oder aber angegeben wird in der Form wie beim Schachbrett die Bezeichnung der Felder. Beispielsweise würde eine solche Stelle im dargestellten Falle genügend bezeichnet sein mit den Buchstaben

$$
\text { f. } 1 \text {, f. } 2 \text {, s. } 3 \text {, s. } 4 \text {, h. } 1 \text {, h. } 2
$$

von links gerechnet als das fünfte Stück zwischen dem 3. und 4. Sagittalschnitt und dem 1. und 2. Horizontalschnitt.

Bezeichnung f. 1, f. 2, s. 3, s. 4, h. 1, die genügend klar das darüber an der Oberfläche liegende Gehirnstïck bezeichnet.

Zur bequemen Orientierung auch für die wörtliche Darstellung ist dann hier noch die Möglichkeit gegeben, auf den Photographien die Hirnwindungen, wie es hier für die Zentralwindungen geschehen ist, durch stärkere Hervorhebung zu bezeichnen. 